\title{
B. P. Hașdeu ou le cercle de l'âme
}

\section{Dorin Ştefănescu}

\section{OpenEdition \\ Journals}

Édition électronique

URL : https://journals.openedition.org/cher/10003

DOI : $10.4000 /$ cher.10003

ISSN : 2803-5992

\section{Éditeur}

Presses universitaires de Strasbourg

\section{Édition imprimée}

Date de publication : 1 décembre 2013

Pagination : 11-28

ISBN : 978-2-86820-560-5

ISSN : 1968-035X

Référence électronique

Dorin Ştefănescu, «B. P. Hașdeu ou le cercle de l'âme », reCHERches [En ligne], 11 | 2013, mis en ligne le 08 février 2022, consulté le 09 février 2022. URL : http://journals.openedition.org/cher/10003 ; DOI : https://doi.org/10.4000/cher.10003

\section{c) (i) (2) (2)}

Ce(tte) œuvre est mise à disposition selon les termes de la Licence Creative Commons Attribution Pas d'Utilisation Commerciale - Partage dans les Mêmes Conditions 4.0 International. 


\title{
B. P. Hașdeu ou le cercle de l'âme
}

\author{
DORIN ŞTEFĂNESCU \\ Université « Petru Maior », Târgu-Mureș, Roumanie
}

\begin{abstract}
T'interprétation proposée examine la spéculation de B.P. Hasdeu sur la Ldestinée de l'âme selon ses deux volets: d'une part la dialectique du moi et de l'inconscient, de l'autre le problème du libre arbitre et de la Providence. Il s'agit d'une véritable phénoménologie spiritualiste qui développe une théorie originale de l'inconscient, en rapport dialectique avec la définition du Moi et son statut intégré à la doctrine de la transmigration et de la réincarnation.
\end{abstract}

\section{Dialectique du moi et de l'inconscient}

Distinguant entre l'organisme du corps (sous-organisme) et l'organisme de l'âme (sur-organisme), les subordonnant à la limitation et respectivement, à l'illimitation, "les deux premières grandes conséquences de l'infini", Hasdeu remarque:

Les autres deux conséquences, directement subordonnées aux premières, sont les deux formes communes de la vie organique terrestre: la veille, où l'être est si limité qu'il peut oublier qu'il est âme; et le sommeil, où il s'illimite toujours jusqu'à ne plus sentir qu'il est corps. (Hasdeu 1991: 105)

Il en résulte deux séries antinomiques: limitation/veille/sous-organisme versus illimitation / sommeil / sur-organisme. Pendant la veille, l'être n'est que corps, dans l'oubli de l'âme qui dort celée dans la forme corporelle; tandis que pendant le sommeil, l'âme s'éveille à une nouvelle vie, se souvenant de son origine illimitée, ignorant à son tour le corps endormi. ${ }^{1}$

1 «L'activité psychique et corporelle sont dans une proportion inverse; pour la psyché l'intervalle d'activité maximale est celui où le corps dort ou, selon Aristote, où il est sur le seuil de la mort» (Dodds 1983: 165). 
La phénoménologie spiritualiste de Hasdeu pose - au niveau de la conscience de soi - les implications de la situation de l'âme incarnée, «temporairement enchaînée au corps» (Hasdeu 1991: 209).² Disons d'emblée qu'en étroite liaison avec cette problématique Hasdeu développe une théorie originale de l'inconscient. Celui-ci comporte trois états, en fonction des déplacements du binôme antinomique limitation/veille vs. illimitation / sommeil :

L'inconscient étant seulement de passage dans un corps, dont il s'illimite totalement par la mort du corps et s'illimite partiellement par le sommeil et d'autres degrés de torpeur, il s'ensuit donc pour lui trois états possibles:

a) L'inconscient limité complètement dans le corps, comme dans la veille commune, quand ce n'est que l'uni-conscience qui est à l'œuvre en l'homme;

b) L'inconscient limité dans le corps, qui sans lui serait mort, mais en même temps se limitant partiellement pour se manifester aussi au dehors du corps, comme dans les cas de télépathie;

c) L'inconscient s'illimitant totalement du corps, qui reste mort, tandis que celui-là devient ce qu'on entend par Esprit. (Hasdeu 1991: 147)

Ce qui nous intéresse pour le moment ce sont les deux premiers cas, et surtout la situation intermédiaire entre a) et b): «Entre $a$ et $b[\ldots]$ il n'est pas facile parfois de s'assurer si l'homme dort ou veille et s'il ne dort et veille éventuellement en même temps» (Hasdeu 1991: 147).

La veille est donc l'état de la conscience uni-dimensionelle, orientée selon la raison vers le monde corporel; en ce sens il y a un rapport de synonymie entre la conscience et la rationalité. D'autre part, l'inconscient n'est l'irrationnel que dans la situation où il est limité dans l'individualité d'un corps et seulement en état de veille (a). L'inconscient est rationnel dans le sommeil, par exemple lorsqu'il est interprété comme un premier niveau de l'illimitation (b), ou dans le cas où, par la mort du corps, l'âme regagne l'illimitation totale (c). Dans cette dernière acception, le surorganisme de l'âme (l'ipséité devenue esprit) dépasse l'état d'inconscience

2 En ce qui concerne la captivité de l'âme dans le corps et le rapport entre psyché et soma (y compris la relation soma / sema, le corps-tombeau), il est intéressant de constater, avec E. R. Dodds, que dans la conception homérique «l'âme n'était pas un prisonnier récalcitrant du corps; elle était la vie ou l'esprit du corps et s'y sentait chez elle». Mais dès l'orphisme - et ensuite dans toute la tradition pythagoricienne, platonicienne et néoplatonicienne la nouvelle mentalité religieuse, «dotant l'homme d'un 'moi' occulte d'origine divine et instaurant le conflit entre l'âme et le corps, [...] a introduit dans la culture européenne une nouvelle interprétation de l'existence humaine» (Dodds 1983: 165). Voir Platon, La République, 588e; Gorgias, 493d; Cratylos, 400b-c; Phaidon, 62b, 81e, 92a; Phèdre, 250c. 
pour acquérir une rationalité plénière: «le sur-organisme, loin d'être inconscient, est plus que conscient, il est bi-conscient, tri-conscient, pluriconscient» (Hasdeu 1991: 142). Il en dérive la conception sur la rationalité de l'âme et l'irrationalité du corps; mais l'âme ne peut acquérir sa véritable rationalité (l'inconscient illimité ou le pluri-conscient de son sur-organisme) qu'en échappant à la veille uni-consciente du sous-organisme corporel, qu' «indépendant du corps ou franchissant les obstacles corporels » (Hasdeu 1991: 136).

Que signifient ces «obstacles corporels»? Ils représentent les limites qui s'accumulent autour de l'âme enchaînée, ces «limites au-dessus des limites» qui restreignent chacune de son côté les qualités de la présence divine dans l'âme:

L'obscurité est la limite de la lumière, le mal est la limite du bien, la laideur est la limite de la beauté, le mensonge est la limite de la vérité [...], tout 'non' est une limite jusqu'à laquelle peut avancer le 'oui', n'est qu'une limite, une nécessité de la nature limitée des mondes et des êtres de l’univers, et disparaît là où il n'y a pas de limites (Hasdeu 1991: 53).

Cette «illimitation entravée» (ou «illimitation soumise à l'entrave») est en fait l'obstacle ontologique nécessaire (dans la conception plotinienne le mal étant nécessaire car il faut que le bien ait son contraire), ${ }^{3}$ représenté par la négation corporelle (ou par celle de la «nature» tout simplement, vue en tant que non-moi) qui s'oppose par sa matérialité même à toute affirmation authentique de l'âme. ${ }^{4}$

\section{Selon Hasdeu, le concept de l'inconscient}

est assez propre si l'on ne recherche pas le sur-organisme en soi, car en soi ce sur-organisme est pluri-conscient, mais l'on regarde seulement du point de vue du corps humain. Pour le corps humain, c'est-à-dire pour notre cerveau uni-conscient, le grand contenu du sur-organisme reste inconnu. Nous employerons donc 'l'inconscient' chaque fois qu'il s'agira précisément du contenu inconnu du sur-organisme (Hasdeu 1991: 143).

Pris en soi, le sur-organisme de l'âme est pluri-conscient, tandis que le sous-organisme du corps est uni-conscient, chacun ayant comme propres (en soi) des manifestations de la conscience à certains niveaux de son développement. La différence consiste dans le contenu connu du corps,

3 «Les maux doivent exister, puisqu'il faut qu'il existe un contraire pour le bien» (Plotin, Les Ennéades, I, 8, 6). Voir Platon, Theaitetos, 176a.

4 «Une âme devient laide à cause de ce mélange et de cette fusion et cette soumission au corps et à la matière» (Plotin, Les Ennéades, I, 6, 5). 
d'un côté, et le contenu inconnu de l'âme, de l'autre côté. À partir d'une perspective corporelle, l'âme reste un inconnu, "le pluri-conscient latent" gardé «celé» dans l'enveloppe corporelle. ${ }^{5}$ C'est comme si le gardien de la prison (pour reprendre l'analogie) ne connaissait pas le détenu que celle-ci abrite entre ses murailles. Bien qu'elle soit enfermée dans le contenu du corps, contenue dans la prison corporelle, l'âme reste en fait non contenue et incomprise en soi; inconnue par le corps, non donnée à la conscience comme existant dans les limites de celui-là, elle représente un inconscient par rapport au corps conscient seulement de soi. Aspect qui ne porte pas atteinte à la rationalité de l'âme; n'ayant pas conscience de l'âme en soi, le corps ne peut «comprendre» cette présence que par le filtre voilé de l'irrationalité de sa conscience limitée. Par conséquent, en soi, l'âme est pluri-consciente et rationnelle; en soi, le corps est uni-conscient et irrationnel; mais pour le corps, l'âme est un contenu inconscient et irrationnel, et cela parce qu'il ne la connaît pas dans son essence. ${ }^{6}$

Il est tout à fait évident que dans une telle conception, l'irrationalité de l'âme bénéficie d'un significatif changement de signe; d'une âme souillée et troublée par l'ignorance maléfique du corps avec lequel elle est obligée de cohabiter, elle devient "source ou canal d'une compréhension intuitive» (Dodds 1983: 244), contexte dans lequel «la compréhension rationnelle cède le pas à une autre instance qui assumera de son propre chef la direction des opérations» (Jung 1990²: 246).7 Pour conclure, dans des phénomènes comme le rêve ou «la folie sacrée", l'inconscient aspire à se transformer en pluri-conscient, et l'irrationnalité à approcher son origine rationnelle. En ce sens, on pourrait parler de l'irrationnalité de la raison, dans le cas où, selon Jung, «la raison bascule dans l'absurde lorsqu'elle se dépare du cœur» (Jung $\left.1990^{2}: 218\right)$.

5 Il s'agit de ce «moi occulte» pythagoricien, que Platon va identifier avec la psyché rationnelle.

6 «Nous pensons encore avec complaisance que notre conscience est la raison, et que notre inconscient est la déraison» (Jung $1990^{1}$ : 178-179). Danger généralement valable, signalé par Jung: "Il est grand temps que l'humanité prenne conscience de ce que l'âme est en réalité, car il se révèle peu à peu, avec une évidence sans cesse croissante, que les plus grands dangers qui menacèrent l'homme de tous temps proviennent de son psychisme, de ce recoin de notre univers empirique jusqu'à présent le plus inconnu de nous» (Jung $1990^{2}$ : 231). Malgré l'apparente singularité de la théorie hasdeienne de l'inconscient, il faut remarquer qu'elle s'apparente aux interprétations jungiennes sur les phénomènes occultes (voir Jung 1990²: 191-248).

7 «La psyché inconsciente a une extension dont nous ignorons l'ampleur, et elle est probablement porteuse d'un sens plus vaste que celui de la conscience» (Jung 1990²: 225). 
Cette théorie de l'inconscient se trouve en rapport dialectique avec la définition du Moi et son statut lié à la doctrine de la transmigration et de la réincarnation. En effet, Hasdeu accepte l'idée de la métempsycose dans son essence: après sa sortie du corps mortel, l'âme entre dans une série de réincarnations succesives. Selon les mentalités traditionnelles, «la mort est [...] le retour à un état primordial et parfait, perdu périodiquement par la réincarnation de l'âme» (Eliade 1978: 193). Ce noyau doctrinaire, il le doit sans aucun doute aux idées religieuses anciennes. Pour le reste, il s'en sépare quant aux points suivants: il n'approuve pas la réincarnation de l'âme humaine dans un corps d'animal ou de plante (ce qu'on appelle métempsycose regressive), mais seulement dans des corps de plus en plus "perfectionnés» sur l'échelle de l'évolution (métempsycose graduellement progressive), 8 thèse influencée par le darwinisme (intéressante, et paradoxale à la fois, cette conception positiviste greffée sur le tronc des doctrines mystiques, "performance» spécifique d'ailleurs à l'éclectisme romantique, bien que Hasdeu considère que «l'éclectisme n'est jamais la destinée des esprits créateurs »). ${ }^{9}$ Par conséquent il ne peut accepter ni la réincarnation comme châtiment de l'âme; par contre, dans la série de ses transmigrations, l'âme se sépare de plus en plus du monde matériel, devient meilleure, brise ses limites, aspect qui tient à la nécessité profonde de la nature, à la loi du devenir universel. Dans ce contexte, la mort est valorisée au maximum: on meurt afin d'accéder à un niveau d'existence obligatoirement supérieur à celui que l'on a quitté dans une existence antérieure. ${ }^{10}$ Enfin, la métempsycose ne représente pas pour Hasdeu une limitation dans une forme de vie inférieure,

8 Pour l'homme, dit-il, il n'y a pas «de fatale bestialité. En aspirant, il arrive plus ou moins, en proportion avec le degré de l'aspiration et l'intensité de la résistance extérieure », ayant la possibilité de s'opposer à la fatalité naturelle (Hasdeu 1984: 533). Il n'est pas étonnant, dans ce contexte, que Hasdeu se considérait un "ultraoptimiste».

9 "Giambattista Vico», in: Hasdeu 1985: 286. Dans ce contexte, Paul Cornea a raison d'affirmer que Hasdeu «domine les faits et les interprétations avec une désinvolture souveraine, en se servant des uns et des autres pour élaborer une doctrine propre. Ecclectique par la nature diverse des sources de référence, il reste profondément personnel par la manière de les ultiliser» («Studiu introductiv» [«Étude introductive»], in: Hasdeu, 1970: XXXII).

10 Méliorisme qui sous-tend toute la création de Hasdeu, proclamé avec vigueur dans la conférence Noi și voi [Nous et vous], où il souligne que «le mal n'est pas universel et exclusif, que bien des choses sont bonnes, que le bien doit affronter le mal, sans crainte et sans merci, l'homme a un libre arbitre et est responsable s'il persiste dans le mal faute d'aspirer au bien, qu'en fin de compte la victoire n'appartient qu'au bien "; "prédominant dans la littérature culte - ajoute-t-il -, l'optimisme est le maître incontesté dans la littérature populaire», dans la sereine philosophie du conte («Revista nouă», V, 1892; in: Hasdeu 1985: 129). 
une nouvelle prison de la matière, mais une illimitation continuelle qui suppose un trajet ascendant de l'âme, "la possibilité d'aller mieux ou plus vite, la possibilité d'arriver plus haut et plus loin ${ }^{11}$ (aspect similaire avec la conception plotinienne qui prévoyait une ouverture et une ascension, mais sans les implications connexes de ce processus). Le fait que Hasdeu comprend la métempsycose dans son hypostase graduellement progressive signifie que le devenir ascendant de l'âme au cours des réincarnations n'est pas continu ou direct, mais suppose le passage à travers des étapes évolutrices intermédiaires: "Une âme humaine inférieure ne saurait pas devenir âme humaine supérieure sans passer par quelques étapes moyennes» (Hasdeu 1991: 110).

Quelles sont ces "étapes du devenir » que l'âme doit traverser jusqu'à son illimitation totale? Ce sont les espèces consécutives et mortelles à travers lesquelles passe l'individuum, représentées dans la figure ci-dessous par des «verges parallèles», figure qui, selon Hasdeu, «aide à la compréhension de l'évolution pour ainsi dire intra-spécifique» (Hasdeu 1991: 110).

Quelques explications. Les niveaux $a-a, b-b, c-c, d-d$ et e-e représentent les incarnations du moi en diverses espèces, la superposition des espèces au cours du temps; $\mathrm{N}$ est l'individu qui suit les cycles des incarnations et des désincarnations successives (sujet de la métempsycose); les intervalles entre deux niveaux sont appelés «intervalles de désincarnation", quand l'âme, abandonnant le corps entre deux incarnations, est un "sur-organisme de désincarnation». Par conséquent, l'âme individuelle (ou le moi immortel) parcourt les étapes «corporelles» de ces espèces, en s'incarnant chaque fois dans l'une d'elles, suivant une ascension incessante d'espèce en espèce.

Cette ascension est le but de l'individu, sa grande mission, par laquelle il se perfectionne toujours, c'est-à-dire il accomplit la loi de l'évolution; tandis qu'une naissance n'est qu'un moyen pour le 'moi' d'entrer dans une espèce quelconque, d'où par une autre naissance ultérieure il entre plus tard dans une autre espèce (Hasdeu 1991: 110).

Pendant les incarnations (qu'on pourrait noter, conformément à la figure, par $\mathrm{Na}, \mathrm{Nb}, \mathrm{Nc}, \mathrm{Nd}$ et $\mathrm{Ne}$ ) l'âme devient une "personne», terme pris en son sens étymologique, c'est-à-dire persona, jouant le rôle de celui qui est caché sous le masque du corps. ${ }^{12}$ Dans les intervalles de désincarnation,

11 Hasdeu 1984: 539 .

12 "Originairement, la persona désignait le masque que portait le comédien, et qui indiquait le rôle dans lequel il apparaissait. [...] En d'autres termes, la persona n'était que le masque d'un assujettissement général du comportement à la coercition de la psyché collective. 
l'âme délivrée de son masque matériel redevient anima, ${ }^{13}$ principe vital, de plus en plus détaché du corps, en train de s'illimiter toujours davantage mais pas absolument. Ce n'est que dans l'hypostase Ne qu'elle

peut aspirer à l'espèce supérieure de sur-homme; pendant les intervalles de désincarnation entre $a-a$ et $b-b$, entre $b-b$ et $c-c$, entre $c-c$ et $d-d$, entre d-d et e-e, elle n'est qu'un ex-homme, de plus en plus délivré, pourtant pas encore complètement. Dans chaque incarnation 'le moi' devient une nouvelle personne, dans le vrai sens du mot 'persona', qui désigne en latin un 'masque', un 'rôle' joué sur la scène pendant une heure ou plus. Ainsi, le même $\mathrm{N}$ se fait à tour de rôle la personne $\mathrm{Na}$, la personne $\mathrm{Nb}$, la personne $\mathrm{Nc}$, la personne $\mathrm{Nd}, \mathrm{Ne}$; mais, pourtant, il ne cesse jamais, incarné ou désincarné, d'être la même individualité, le même 'individuum' $\mathrm{N}$, se souvenant à chaque désincarnation du répertoire de plus en plus haut des 'rôles' joués dans le passé sur la scène de l'évolution universelle (Hasdeu 1991: 111).

Bref, l'individuum incarné est la persona, désincarné il est l'anima. La métempsycose consiste notamment dans cette chaîne des réincarnations succesives entre deux bouts infinis: l'infini dont l'âme descend en procession pour s'incarner dans le fini de la première persona, et l'infini où l'âme se réintègre par conversion, sans plus s'incarner. ${ }^{14}$

Quel est le rôle de l'inconscient dans le processus de transmigration de l'âme?

Chaque 'moi', individualité ininterrompue dans la chaîne de toutes ses transformations succesives, à chaque nouvelle incarnation fait une nouvelle personne, c'est-à-dire joue un nouveau rôle. Dans ce nouveau rôle le moi a manifestement l'uni-conscience du personnage qu'il joue, tout en gardant célée la pluri-conscience de tous les personnages joués dans le passé (Hasdeu 1991: 93).

Dans sa coexistence avec le corps, l'âme est uni-consciente, tandis que la même âme désincarnée est pluri-consciente. Autrement dit, l'âme-

[...] Comparée à l'individualité du sujet, sa persona n'est qu'une réalité secondaire, un simple artifice, un compromis à la constitution duquel d'autres participent souvent bien davantage que l'intéressé lui-même. Sa persona n'est qu'une apparence et, pourrait-on dire par boutade, une réalité à deux dimensions» (Jung 1989: 83, 84).

13 C'est, selon Jung, l'attitude intérieure ou l'âme inconsciente, opposée à l'attitude extérieure consciente de la persona. Voir Jung 1989: 153-160. La persona, souligne à son tour Jean Haudry, désignait dans l'ancienneté l'individualité physique, «la notion de personne était tournée vers l'extérieur» («De l'être social à l'âme individuelle dans la tradition indo-européenne», in: Shishmanian A. A., Shishmanian D. 2006: 127).

14 «Chaque 'moi' représente une multitude d'espèces, par lesquelles il était déjà passé, sauf celles par où il passera encore. On pourrait dire que chaque 'moi' constitue par lui-même une immense espèce, tendue de l'infini et aspirant à l'infini» (Hasdeu 1991: 109). 
persona, influencée par le sous-organisme corporel, est uni-consciente, et l'âme-anima, illimitée dans le sur-organisme, est pluri-consciente. Dans la perspective corporelle ( $i$. e. du point de vue du rôle que l'âme incarnée joue sur la scène de la matière, comme persona), l'âme est «le pluri-conscient latent», caché derrière le masque. L’inconscient est, par conséquent, justement la virtualité existentielle de l'âme, qui contient - en somme - toutes les consciences des existences antérieures, mais aussi la pluri-conscience qui traverse non modifiée la série des réincarnations. "Manifestement», l'âme est persona, présentant dans l'actuel l'uni-conscience du personnage corporel. "Inapparemment», l'âme est anima, gardant virtuellement la pluri-conscience de sa nature divine. L'inconscient est donc le contenu inconnu de ce pluri-conscient latent (devenu esprit dans les intervalles de désincarnation), caché dans sa propre identité de soi, tandis que le conscient est ce qui est connu en tant qu'uni-conscient manifesté dans la diversité que suppose chaque incarnation à part:

En ce qu'on appelle 'l'inconscient' se trouvent donc non pas seulement toutes les consciences des existences humaines antérieures de l'individu, mais aussi des couches d'instinct et d'impulsion des existences zoologiques, botaniques, peut-être même minéralogiques, tandis que ce qu'on appelle 'conscience', c'est-à-dire ce qu'on devrait appeler 'uni-conscience', n'appartient qu'à la seule existence humaine actuelle de chacun (Hasdeu 1991: 146). ${ }^{15}$

L'inconscient est intégré de la sorte dans la doctrine de la métempsycose.

«Par la loi de l'evolution universelle, chaque 'moi' doit se développer de bas en haut à travers plusieurs niveaux du devenir»(Hasdeu 1991: 109), théorie dont découlent deux conséquences: d'une part, l'immortalité du 'moi' et non de l'espèce (qui est mortelle), et, d'autre part, le fait que «chaque 'moi' constitue par lui-même une immense espèce, tendue de l'infini et aspirant à l'infini» (Hasdeu 1991: 109). Les espèces ne représentent que les formes des incarnations temporaires (et temporelles) du moi; ce n'est qu'à travers ces manifestations dans le visible (en tant que persona) qu'il peut se développer suivant le trajet ascendant du devenir, $i$. e. de l'illimitation vers l'infini (en tant qu'anima). Changeant, au cours des transmigrations, les masques des espèces succesives, le moi apparaît comme une sorte d'espèce

15 Un nouvel exemple de cette greffe "exotique» de la métempsycose sur le tronc du positivisme darwiniste: "J'ai toujours été darwiniste - avoue Hasdeu -; c'est-à-dire depuis toujours, même avant Darwin, je pensais que l'homme n'est autre chose que la plus haute manifestation terrestre d'une même énergie qui, à des niveaux de plus en plus bas, se présente à nous en d'autres animaux, plantes, et même en des cristaux» («Sarcasm și ideal» [«Sarcasme et idéal»], in: Hasdeu 1985: 202). 
éternelle soumise aux métamorphoses, mais subordonné dans la profondeur de son existence au principe vital qui fonde le progrès universel. Les espèces peuvent être considérées des limites nécessaires au moi dans des moments différents de son évolution. Un principe de l'avancement caractérise le mécanisme du devenir, avancement inconcevable sans une connaissance anamnétique progressive, d'autant plus profonde qu'elle récupère les expériences assumées dans les incarnations antérieures, «dans la totalité non divisée du sur-organisme individuel» (Hasdeu 1991: 147). ${ }^{16}$ Ce que Hasdeu appelle «l'imperceptibilité de l'avancement» est l'expression d'un rapport dialectique entre le conscient et l'inconscient, rapport qui a comme protagoniste principal le moi. Cet avancement est

une action de l'inconscient qui dépose dans la conscience beaucoup ou peu, peu ou beaucoup, combien il peut et combien il faut, c'est-à-dire: 1 . Combien il peut lui-même selon son propre niveau d'avancement dans l'évolution; 2. Combien il faut à l'acteur dans son nouveau rôle, pour qu'il ne soit ni embrouillé par trop, ni entravé par trop peu (Hasdeu 1991: 147).

Il est évident maintenant que par le concept d'inconscient Hasdeu a en vue un set d'informations, plus exactement un flux informationnel caché dans la profondeur du moi. Dispositif qui n'a rien à faire avec l'inconscient collectif, dans son acceptation jungienne, car il s'agit d'une dot individuelle, accumulée au cours des réincarnations succesives d'une seule âme. Pour Hasdeu - comme on a déjà vu - le moi n'est autre chose que l'âme, soit en son hypostase incarnée dans le sous-organisme corporel («le moi temporairement enchaîné au corps»), soit décorporalisée dans le sur-organisme spirituel ou pneumatique («le moi caché sous l'écorce, dans l'écorce et au-dessus d'elle») (Hasdeu 1991: 209, 138). ${ }^{17}$ L'illimitation

16 «En chaque homme, 'le moi' passe comme personne mortelle par le sous-organisme pondérable du corps, mais ne vit comme individualité immortelle que par le surorganisme impondérable de l'âme» (Hasdeu 1991: 136), de sorte qu'au niveau du dernier intervalle de désincarnation il devient "une mémoire intégrale de l'existence intégrale», le garant de son identité et de son unité: "Une individualité, un 'moi' se fonde sur la mémoire, car ce n'est que par son entremise que quelqu'un peut apprendre qu'il est l'un et le même dans une longue série de moments, qu'elle lie en une seule unité» (Hasdeu 1991: 93). En ce qui concerne l'anamnesis, ce par quoi Platon entend «le souvenir des choses que notre âme a vues jadis» (Phèdre, 249c), il ne désigne pas les incarnations antérieures, mais «la réalité vraie» des Idées. Ce qui ne veut pas dire que la mémoire de type pythagoricien n'apparaît chez Platon aussi, par exemple dans le mythe d'Er dans $L a$ République (620c), où ceux qui se préparent pour une nouvelle incarnation gardent «le souvenir des malheurs d'avant», appartenant à une vie antérieure.

17 À l'encontre de la conception homérique, où 'le moi' est corps et âme à la fois, hypothèse selon laquelle il y a dans l'homme une sorte d'existant étranger, un double, un deuxième moi plus inconscient, la psyché. Louis Graz souligne d'ailleurs l'unité individuelle de la 
représente donc justement l'épreuve continue de libérer les informations déposées dans l'âme, ce qui permet à celle-ci d'approcher de plus en plus l'infini dont elle s'est détachée dans sa chute. La quantité des informations que l'inconscient transmet au conscient dépend du "niveau d'avancement", c'est-à-dire du degré évolutif de l'âme sur l'échelle de son devenir, et aussi de la nécessité qu'elle manifeste dans un moment donné de l'évolution. Nécessité qui va de pair avec la mesure qui règle le flux d'informations qui déversent dans le conscient, conformément aux exigences d'une âme consciente du rôle qu'elle doit accomplir en ce monde. ${ }^{18}$ En conclusion: l'inconscient dépose dans la conscience «combien il peut et combien il faut».

\section{Du Libre arbitre et de la Providence}

La théorie hasdeienne du moi, greffée sur le rapport dialectique entre le conscient et l'inconscient, ne saurait être comprise dans sa complexité réelle que par l'appel au concept de libre arbitre et au concept complémentaire de fatalité.

En chaque nouvelle existence l'homme sent consciemment son libre arbitre et inconsciemment une fatalité qui le pousse en avant. Le libre arbitre conscient appartient au présent personnel; la fatalité inconsciente est la ligne tracée depuis le début à travers tout le passé individuel. Si j’ai déjà été A', A", $\mathrm{A}^{\prime \prime}$, il faut fatalement que je devienne A"'", et pas autre chose, ni B, ni C; mais dans le cercle de cette fatalité inconsciente de ne pouvoir être rien que A, je bénéficie d'un libre arbitre personnel conscient de rester sur place, capable de me remettre, de me faire progresser, de m'élever avec plus de succès dans ma sphère individuelle A. Dans l'image d'une seule vie, sans un enchaînement de plusieurs incarnations l'une après l'autre, le libre arbitre conscient du moi n'a aucun sens, et la fatalité inconsciente du moi non plus, car la fatalité individuelle du présent est la somme de tous les libres arbitres personnels du passé. (Hasdeu 1991: 146)

Dans l'existence limitée du corps, le moi (ou l'âme) semble mener une double vie. D'une part, il est conscient de sa capacité d'influencer son devenir, reconnaissant en soi la présence du libre arbitre. D’autre part,

personne dans l'Iliade, l'homme homérique ayant également "corp» et «âme» (Graz L., 1960, «L'Iliade et la personne», Esprit, sept.: 1390-1403). Voir aussi Nédoncelle M., 1948, «Prósōpon et persona dansl'antiquité classique», Revue des sciences religieuses, 22:277-299.

18 Elle doit «savoir choisir la vie moyenne», choisissant «sagement», en toute connaissance, entre les extrêmes limitatives ( $c f$. Platon, La République, 619a). Et cela, précise Hasdeu, «car ce n'est que l'absolu dont je suis capable qui est infini» («Legea infinitului» [ L La loi de l'infini»], Cuvântul, 1, 1906; in Hasdeu 1985: 133). 
au-delà de cette conscience du présent, du temps qui mesure l'existence corporelle, le moi sent inconsciemment un flux intérieur qui l'emmène avec soi, le transcende en tant qu simple moi conscient, s'imposant à lui en tant que fatalité transcendentale. La fatalité est perçue comme un écho lointain de l'illimitation, matrice personnelle inaliénable qui inscrit le moi, au-delà de sa volonté, sur la voie du devenir ascendant. Il faut souligner le caractère individuel du libre arbitre et surtout de la fatalité, qui n'implique que le moi seul dans les avatars de ses hypostases. ${ }^{19}$ Le moi est ce qu'il a été, car les vies passées décident en grande mesure quant à la vie actuelle, la fatalité préexistant à toute incarnation, les surplombant toutes, les renfermant dans leur série illimitée. Mais il est aussi ce qu'il choisit d'être, au-delà de la fatalité qui porte ses pas sans qu'il le sache, parce que «nous avons le pouvoir de choisir; c'est à nous de choisir soit le bien, soit le mal, selon notre volonté ».20 Mais l'âme ne peut savoir ce qu'elle veut que limitée par le corps mortel; ce n'est qu'ici que le moi est conscient du rôle qu'il a à jouer. ${ }^{21}$ Et cela puisque le libre arbitre ne peut pas être conçu en dehors de la conscience; comme tel il n'existe que dans le moi incarné en chaque nouvelle existence. Déterminé par une virtualité bénéfique inconsciente, enfermé dans «le cercle de cette fatalité inconsciente» conformément à laquelle il ne peut être que ce qu'il a été, le moi-âme a pourtant à sa disposition la volonté non pas de changer la voie, "la ligne tracée depuis le début à travers tout le passé individuel», mais de s'élever «avec succès» dans sa sphère individuelle, de s'illimiter de plus en plus, d'aspirer intentionnellement vers l'infini, l'intention étant justement l'horizon du libre arbitre: «En aspirant, il arrive plus ou moins, selon le degré de l'aspiration et l'intensité de la résistance du dehors; mais il n'y arrive pas sans aspirer, maître comme il l'est d'aspirer ou non » (Hasdeu 1984: 539).

Par conséquent, dans la limite d'une seule incarnation, le libre arbitre et la fatalité manquent de sens; leur sens ne se manifeste que dans le cycle des

19 Un moi qui se soumet, comme dans la conception stoïque, à la loi universelle du monde, qui est la divinité, se trouve entre les mains du "destin» (peproméne, heimarméne) qui le dirige et qui détermine l'ensemble de la vie et, implicitement, l'existence de tous les vivants. Il a quand même le pouvoir et le devoir d'une libre décision, la responsabilité de ses choix et de ses faits (voir Rohde 1985: 384-385).

20 Corpus Hermeticum, Divers fragments hermétiques, I; voir aussi Livre XII, Le Cratère ou sa monade, 30-33. "La faute est à celui qui choisit, le dieu est innocent», proclame Platon dans La République, 617e. Sur l'importance du choix (diairesis) et la différence entre le platonisme et l'orphisme à cet égard, voir Platon, La République, 618a-620e.

21 Comme dans le zurvanisme: «dans le monde matériel, ce n'est que l'homme qui dispose du libre arbitre. Mais l'âme ne peut agir que par le corps qu'elle habite» (Eliade 1978:311). 
transmigrations, sur la voie qui parcourt toutes les réincarnations succesives. C'est la raison pour laquelle la fatalité individuelle du présent est la somme de tous les libres arbitres personnels du passé, le libre arbitre conscient du moi n'étant qu'un chaînon nécessaire et essentiel dans la grande chaîne de la fatalité inconsciente. Spécifique à l'homme, le libre arbitre est pourtant plus que la volonté de s'élever vers l'illimitation; il représente l'action consciente par laquelle l'âme se récrée soi-même, la création continuelle par laquelle le moi «croît» en liberté, «inspiré » par une pulsion intérieurement enrichissante :

Face à l'espèce supérieure, l'homme garde la totalité de sa volonté, la plénitude de ce libre arbitre qu'il ne perd même pas devant Dieu, vu que, créé par Dieu par une limitation de l'infini, il ne se crée pas ensuite seulement lui-même à son tour par l'illimitation vers l'infini, mais se crée notamment sachant qu'il se crée, non dans l'ignorance comme les êtres inférieurs, mais ayant la conscience et la responsabilité de sa propre création, ce que nous entendons par le libre arbitre. (Hasdeu 1991: 65)

Grâce au libre arbitre l'âme est plus que consciente; elle est avant tout conscience de soi (daimon), son œuvre de ré-création représentant un acte de restauration réalisé en pleine responsabilité. C'est pourquoi l'âme se crée sachant qu'elle se crée, ayant la responsabilité de sa propre création et, implicitement, de sa propre liberté. C’est justement ce que Hasdeu entend par libre arbitre.

D’autre part, la fatalité (terme plus ou moins impropre, générateur de malentendu), dans la conception de Hasdeu, n'est pas un déterminisme aveugle, une providence intolérable, mais une maîtrise divine, une surveillance créatrice. La doctrine de la fatalité inconsciente ne mène pas au fatalisme moral, qui ne peut être que celui des limites et, au bout du compte, celui de la dernière limite (or, le fatalisme ne saurait cohabiter avec l'affirmation du libre arbitre), mais, par contre, à l'orientation de l'énergie spirituelle en accord avec le devenir ascendant, avec la liberté sans limites. Dans l'acception hasdeienne, la fatalité désigne une certaine limitation, mais guidante, librement consentie par le libre arbitre qui lève toujours le niveau de cette prédestination à des côtes de plus en plus hautes, dans des horizons toujours élargis. ${ }^{22}$ Ce fatum positif ne peut être que la providentia transcendentale, dans le sens qu'elle est préexistante à toute conscience du

22 C'est, comme chez Philolaos, "une sorte de surveillant», une surveillance-providentia dont il ne faut se détacher, ni l'éviter. Voir aussi Platon, Phaidon, 62b (à propos des sens du terme phroura). 
moi incarné dans le présent, et à la fois postexistante à celle-ci, étant en même temps - comprise en tant que grâce divine prédestinée - son donnée originelle fondatrice.

Parlant, dans L'Histoire critique des Roumains, de «la sélection providentielle» («le doigt de la Providence») qui n'est pas moins féconde que la sélection naturelle, Hasdeu affirme:

cette action de la Providence, bien que continuelle, n'impose pourtant pas à l'humanité [...] une direction nécessaire, mais facilite seulement sa possibilité d'avancer mieux ou plus vite, la possibilité d'arriver plus haut ou plus loin, la possibilité de lutter avec succès contre la nature, une simple possibilité, revenant à la responsabilité de l'homme d'accomplir ou de négliger sa mission, de faire plus ou moins, de se lever ou de tomber. (Hasdeu 1984: 539)

Adepte de la théorie de la sélection naturelle proposée par Darwin, Hasdeu emprunte en même temps à Wallace l'idée de la sélection providentielle, en avouant: "J’ai toujours été darwiniste. Darwin a découvert la grande loi de l'évolution de toute l'espèce humaine de l'animal inférieur »; mais "l'homme étant matière et esprit à la fois, la loi de Wallace est une démonstration matérielle du devenir humain sous la direction de la Providence ».23 Si le libre arbitre impose une direction assumée par l'uni-conscience de l'âme dans son hypostase incarnée, la Providence imprime une sur-direction qui soustend et oriente toutes les directions individuelles. La sélection naturelle, par conséquent, n'est pas suffisante pour ouvrir à l'être l'horizon du devenir, pour que l'âme puisse devenir ce qui lui est donné d'être: "une telle incalculable distance entre l'état latent et l'état manifeste, entre être et devenir, ne saurait pas être attribuée à une quelconque évolution matérielle»; il faut "reconnaître dans le devenir de l'humanité l'orientation d'une intelligence suprême, l'action d'une sélection providentielle» (Hasdeu 1984: 531).24

23 «Sarcasm și ideal» [«Sarcasme et idéal»], in: Hasdeu 1985: 22; Hasdeu 1984: 531. «Prenons seulement la sélection naturelle, seulement ce qui est scientifique dans la théorie darwiniste, seulement ce qu'on ne peut pas contester, et voyons si une doctrine si peu spiritualiste n'est pas obligée elle-aussi en fin de compte de reconnaître le doigt de la divinité» (Hasdeu 1984: 518). «Il est évident que l'homme n'avait été créé par la sélection naturelle ou une autre loi physique, ni par la matière inconsciente, mais par une cause intentionnelle, une force intelligente, un pouvoir prévoyant, ayant comme but le progrès humain futur, et non le misérable statu quo du sauvage » (Hasdeu, 1873, «Materia, spiritul și divinitatea» [«La matière, l'esprit et la divinité»], Columna lui Traian, IV, 8: 144).

24 La sélection naturelle n'épuise pas le devenir universel, n'explique pas sans reste la donnée originelle qui guide le destin humain; de sorte qu'il «reste quelque chose en réserve, un petit residuum plus essentiel que tout, un noyau qui ne se résout que par la sélection providentielle» (Hasdeu 1984: 538). 
On revient ainsi au desideratum de la réalisation de cet «organisme de pensées» qui, sous le signe de "la conciliation» philosophique, entrelace la sphère du connu et celle de l'inconnu récognoscible, dans notre cas la voie connue de la sélection naturelle et la voie encore inconnue, mais non incognoscible, de la sélection providentielle, ce qui est reconnaître, pardelà l'évidence des visibles et directement vérifiables, «tout ce qui reste hors de la portée de l'esprit humain», c'est-à-dire «l'action maîtrisante de la divinité», "la Providence étant le fondement sur lequel se dresse tout l'édifice de la réaction de l'homme contre la nature» (Hasdeu 1984: 528): «au-dessus des choses tangibles, se manifeste une force dirigeante touteprésente, inexprimable, qui n'est pas donnée à l'homme de connaître, mais qu'il ne peut ne pas reconnaître»; par conséquent, "ne pas reconnaître la Providence, ne pas laisser à l'homme son libre arbitre envers la nature et la Divinité, c'est ne pas comprendre l'histoire $» .{ }^{25}$ C'est pourquoi, la Nature ou la Providence divine n'écrase pas le libre arbitre, la liberté et la responsabilité de l'orientation, mais les sur-veille dans le sens de leur insertion dans le mouvement ascendant du devenir universel: «La Nature ne tue pas le libre arbitre, n'empêche pas le progrès", (pré)établissant à toutes choses une «direction souvent interrompue et ensuite renouée d'intervalle en intervalle [...], et la surdirection de toutes les directions [...] est dans la providence» (Hasdeu 1984: 292-293).26

La théorie est apparentée à la doctrine plotinienne du libre arbitre. Partant du postulat de la bonté du monde, Plotin insiste sur l'action providentielle bénéfique, la providence étant en fait la préscience divine, l'ordre universel qui oriente tout ce qui est vers un but unique, celui de la perfection:

Toutes les choses doivent dépendre les unes des autres, et 'tout conspire', selon ce qu'on a bien dit, non seulement dans le cas d'une seule chose particulière, mais plutôt [antérieurement, n. n.] au cas de tout l'univers. Le principe unique unifie les parties multiples du vivant et en fait une. ${ }^{27}$

Dans la conception plotinienne, la providence signifie bonté et ordre divins, prévison nécessaire (prévoyance), éternelle et toute-présente, qui - ici également - doit être reconnue comme telle:

25 «Istoria» [«L'histoire»], in: Hasdeu 1985: 21, 19.

26 «La sélection providentielle ne détruit pas le libre arbitre humain et n'écarte pas la responsabilité morale» (Hasdeu 1984: 533).

27 Les Ennéades, II, 3, 7. Jamblique s'exprime dans le même sens: «il n'y a rien dans l'univers qui s'éloigne de l'ordre» (Du destin, V). 
En partant de ce qu'on voit dans l'univers, il faut reconnaître que tel est l'ordre éternel de l'univers, étendu sur toute chose, jusqu'aux plus petites. [...] Par conséquent, ce qui change change, mais ne change et ni ne prend une forme par hasard, mais ainsi qu'il est beau et conforme à être produit par les forces divines. Car tout ce qui est divin produit conformément à sa nature. ${ }^{28}$

Quel est le rapport entre cette providence divine (la fatalité inconsciente, chez Hasdeu) et le libre arbitre du moi conscient? C'est à Plotin de répondre:

L'âme détient la pulsion d'accomplir sa fonction, car l'âme réalise tout, ayant le rôle d'un principe. Soit qu'elle avance tout droit ou par des chemins détournés [...], la justice y suit ses actions. Car l'univers persiste éternellement, conduit par l'ordre et le pouvoir de celui qui le maîtrise. [...] Nous accomplissons les actions naturelles de l'âme jusqu'à notre chute dans la multiplicité de l'univers; dans cette ultime situation, nôtres sont le jugement et la chute même et le mauvais destin dernier. ${ }^{29}$

Quand l'âme s'efforce d'accomplir la fonction qui lui est propre, alors elle suit le chemin juste; quand elle s'égare (en voulant le mal) la justice divine la fait l'esclave de l'ordre physique qui gouverne dans l'univers. Dans les termes de Hasdeu, la fonction propre de l'âme est de suivre le chemin juste de l'illimitation; en s'en écartant, elle s'enferme dans de nouvelles limites. Par conséquent, ce n'est pas la fatalité providentielle qui s'oppose au libre arbitre, que d'ailleurs celui-ci renforce, tout comme la liberté se conjugue avec la nécessité. ${ }^{30}$ La bonne volonté de l'âme est altérée par la matérialité du corps où elle est enfermée, soumise aux vices et aux désirs de la matière. L'âme laide, dit Plotin, est «impure et menée par toute sorte d' attractions vers le sensible, ayant par surcroît mêlée en elle une grande partie des traits

28 Les Ennéades, III, 2, 13.

29 Les Ennéades, II, 3, 8.

30 En ce qui concerne le dépassement de l'antinomie entre la nécessité et le libre arbitre contingent, chez Proclus, voir Libera 1994: 431, n. 102, référence à la prop. 18 des Elementatio theol.). Pour Schelling (Le système de l'idéalisme transcendental, trad. roumaine: Humanitas, București, 1995), le phénomène de l'arbitraire (liberté de la volonté) est le subjectif absolu, qui se manifeste «dans les limites de la finitude», mais en concordance avec une harmonie préétablie. "Bien qu'il existe une prédétermination concernant le moi librement déterminant [...], cette prédétermination ne peut être pourtant conçue que toujours par l'entremise d'un acte originel de la liberté». Cette prédétermination ou harmonie préétablie est une "nécessité cachée » qui fait rapport avec la liberté, "une providence inconsciente qui intervient dans le jeu même de la liberté» ou "un inconscient éternel» comme «racine invisible» des actions librement conscientes, «un esprit unique qui crée en tous» et qui «ne se révèle et ne se dévoile que successivement par le jeu même de notre liberté» (Schelling 1995: 257, 260, 276, 281, 283). 
du corps, cohabitant avec bien des éléments matériels et permettant en elle une idée étrangère, et change pendant ce mélange avec l'élément inférieur ».31

Il persiste pourtant ici une contradiction: si le libre arbitre est ombragé par les penchants de la matière, et que l'âme s'égare sur des chemins détournés qui la lient davantage à l'existence corporelle, quel peut être le rôle de la Providence divine? Hasdeu essaie de résoudre cette contradiction à l'aide du phénomène de la double personnalité, phénomène qui assume en fait le principe des deux concepts contraires de la spéculation grecque. En invoquant «la grande question de plusieurs existences évolutives de chaque individualité» (Hasdeu 1991: 189), il met sur le tapis le cas «bipersonnel» où l'âme incarnée est la scène d'un combat entre ce qu'elle a été dans les existences antérieures (état appelé "condition primaire») et ce qu'elle est (ou tend d'être de plus en plus) dans l'existence actuelle (état appelé "condition seconde»). Si la première condition est dominée par l'enchaînement au corps, penchant vers le mal, la seconde, «souterraine » et inconsciente, oriente l'âme sur la voie juste. Il est sous-entendu que dans la condition primaire le libre arbitre est altéré par une matérialité héritée des incarnations antérieures, inférieures, ${ }^{32}$ anéanti presque quant à sa fonction propre. Le rôle de la condition seconde est justement celui de la fatalité inconsciente, de la providence dirigeante: de régler l'égarement de l'âme et de la ramener sur la bonne voie, celle qui mène vers l'harmonie universelle dont la clé est à Dieu: "voilà ce que nous entendons par Providence. Qu'on ne pense pas que ce pédagogue de l'Univers nierait le libre arbitre individuel ou national, l'unique base de la responsabilité morale de l'homme» (Hasdeu 1984: 358). On assiste à un "phénomène de combat acharné dans le même individu entre deux personnalités antagonistes», à "un conflit entre deux niveaux de l'évolution d'une seule individualité» (Hasdeu 1991: 190).33

La condition humaine se situe sous le signe de la dualité, sous l'action conjuguée de deux maîtres: d'une part, «maître de tendre ou de ne pas tendre", conformément au libre arbitre, l'homme est, d'autre part, soumis à la Providence divine qui, «vu qu'elle nous dirige, ne détruirait-elle pas le

31 Les Ennéades, I, 6, 5.

32 Puisqu'il garde encore «le souvenir des ennuis d'avant» (Platon, La République, 620c).

33 À la rigueur, ce conflit "d'intérêts» exprime l'opposition entre «le remous commun» et «la rotation céleste», mentionnée par Platon dans Phèdre, 248a. Il faut rappeler que pour Hasdeu «la sélection providentielle» ne tue pas «le libre arbitre». Si dotée que soit la paideuma d'un peuple "potentiellement par la sélection providentielle», elle ne pencherait pas vers le bien «si elle ne progressait pas par son propre libre arbitre» («Vladislav Basarab», Columna lui Traian, II, 37, le 4 octobre, 1871: 144). 
libre arbitre humain?» (Hasdeu 1984: 531). Mais l'homme, tout comme les peuples, porte "le sceau de la prédestination», c'est-à-dire il comprend et la condition primaire et la condition seconde, l'accent tombant non pas tant sur la donnée prédestinée de la fatalité providentielle que surtout sur la liberté par laquelle l'homme assume son choix de renouvellement. ${ }^{34}$ Car ce n'est pas «moins palpable la tâche de l'individualité humaine, qui restera figée à jamais si elle ne progresse pas par son propre libre arbitre, si largement qu'elle soit dotée potentiellement par la sélection providentielle» (Hasdeu 1984: 535, 537). L'intention qui déclenche la tension de l'âme vers l'infini, conformément à son libre arbitre, est - à son tour - surdirectionnée par la cause intentionnelle de la providence; en connaissant la première, l'âme doit reconnaître la deuxième, afin de ne pas rester figée, afin d'être une continuelle mise en action. Par conséquent, non seulement le libre arbitre, non pas la seule providence, engagés dans un processus alternant, mais leur simultanéité, vue comme acte circulaire qui reconcilie l'inférieur et le supérieur, le grand Cercle de la détermination processive du «bas» limité et la sur-détermination conversive du «haut» infini:

Sans Divinité, la civilisation se tiendrait trop bas; sans libre arbitre, elle se tiendrait trop haut; la simultanéité de la Divinité et du libre arbitre reconciliant seule dans l'histoire le haut extrême et le bas extrême; quant aux points intermédiaires, ils dérivent de la pression des circonférences, car la Providence ne change pas les rapports des choses..$^{35}$

Ainsi, conformément à la suprême loi de l'évolution, «l'homme nouveau » arrive à remplacer «l'homme ancien », l'état premier n'étant qu' « un fragment, un reste de personnalité entière d'une autre vie antérieure moins parfaite» (Hasdeu, 1991: 190), tandis que dans l'état second l'âme atteint un niveau

34 Un équilibre pareil, entre la liberté (spirituelle) et la fatalité (matérielle) revalorisée, est envisagé - bien que dans un contexte philosophique tout à fait différent - par I. Heliade Rădulescu. Vu l'inertie et le passif qui définissent sa structure, la matière est soumise à l'acte prédestiné d'ordre spirituel, à "sa fatalité, à laquelle elle ne saurait pas échapper», fatalité nécessaire sans laquelle "rien ne serait sûr, tout serait hasard» (Heliade, 1859-1869: 269). Le fatum matériel représente la certitude prédestinée à la matière, qui la rend nécessaire à la liberté de l'esprit dont l'acte créateur peut éviter l'influence néfaste (réellement «fatale») du hasard. Le fait que la matière ne peut pas échapper à la fatalité n'est donc pas un mal nécessaire mais, au contraire, sa loi destinale qui lui offre le droit et le devoir de s'avérer indispensable à la création de l'équilibre universel. Chez Heliade, la fatalité est «un état de passivité, d'inertie, auquel on ne saurait pas échapper ou attendre, sans que l'on s'y oppose, ce qui lui viendra de l'être actif ou libre» (Heliade 1892: 29).

35 «Originea civilizațiunii» [«L'Origine de la civilisation»], Columna lui Traian, IV, 9, 1873; apud Eliade 1987: 102. Mais, comme dans toute sélection, pas tous seront élus: «Nous ne mourrons pas tous, mais tous nous changerons» $(1$ Cor. 15, 51). 
supérieur dans son effort d'illimitation. C'est l'un des arguments qui, au-delà du fait de plaider en faveur de la métempsycose graduellement positive, confère à la doctrine de Hasdeu un profond optimisme anthropologique.

\section{Bibliographie}

Dodds E. R., 1983, Les Grecs et l'irrationnel; trad. roumaine: Dialectica spiritului grec, București, Meridiane.

Eliade M., 1978, Histoire des croyances et des idées religieuses, II, Paris, Payot.

Eliade M., 1987, Despre Eminescu și Hasdeu [Sur Eminescu et Hasdeu], Iași, Junimea. Hasdeu B. P., 1970, Etymologicum Magnum Romaniae, I, Bucureşti, Minerva.

Hasdeu B.P., 1984, Istoria critică a românilor [L'Histoire critique des Roumains], București, Minerva.

Hasdeu B.P., 1985, Scrieri filosofice [Écrits philosophiques], București, Ed. Științifică și Enciclopedică.

Hasdeu B.P., 1991, Sic cogito, Craiova, Scrisul Românesc.

Heliade Rădulescu I., 1859-1869, Equilibru intre antithesi sau Spiritul și Materia [L'Équilibre entre les antithèses ou l'Esprit et la Matière], București.

Heliade Rădulescu I., 1892, Historia critică universală [L'Histoire critique universelle], București.

Jung C. G., 1989, Dialectique du Moi et de l'inconscient, Paris, Gallimard.

Jung C. G., 1990' Essai de l'exploration de l'inconscient, Paris, Denoël.

Jung C. G., 1990², L’Âme et le Soi. Renaissance et individuation, Paris, Albin Michel.

Libera A. de, 1994, La mystique rhénane. D’Albert le Grand à Maître Eckhart, Paris, Seuil.

Rohde E., 1985, Psyché; trad. roumaine, Bucureşti, Meridiane.

Schelling F.W.J., 1995, Le système de l'idéalisme transcendantal; trad. roumaine: Bucureşti, Humanitas.

Shishmanian A. A., Shishmanian D. (éd.), 2006, Ascension et hypostases initiatiques de l'âme. Mystique et eschatologie à travers les traditions religieuses, t. I, Actes du Colloque International d'histoire des religions «Psychanodia», Paris, INALCO, 7-10 septembre 1993. 